\title{
ÉTICA EN LA INVESTIGACIÓN Y LA PRÁCTICA CLÍNICA: UN BINOMIO COMPLEJO
}

\author{
ETHICS IN RESEARCH AND CLINICAL PRACTICE: A COMPLEX PAIRING
}

Alberto Córdova-Aguilar 1,2,a

\begin{abstract}
RESUMEN
La investigación en salud es vital para el avance de la ciencia y el desarrollo de un país. Sin embargo, investigar con ética en la práctica clínica diaria es un proceso lento. Diversos factores juegan un rol crítico al inicio de cualquier investigación: el centro laboral, el momento adecuado, los recursos con los que se cuenta, la cantidad de sujetos de estudio que se podría involucrar y hasta el entorno sociofamiliar propicio del investigador. Todos estos factores están relacionados de forma directa o indirecta con problemas éticos entre el médico investigador y el paciente o sujeto de investigación; más aún en países como el Perú, donde el bajo nivel educativo de la población acrecienta cierta postura de poder del médico. Asimismo, la aparición de nuevas áreas en la Medicina como los cuidados paliativos, la reproducción humana asistida, la terapia genética y la ingeniería de tejidos hacen aún más complejo este binomio.
\end{abstract}

Palabras clave: Investigación médica; Bioética; Relación médico paciente. (fuente: DeCS BIREME)

\begin{abstract}
Health research is vital for the advancement of science and development in a country. However, researching with ethics in daily clinical practice is a slow process. Several factors play a critical role at the beginning of any investigation: the workplace, the right time, the resources with which it is counted, the number of study subjects that could be involved and even the socio familiar environment of the researcher. All these factors are directly or indirectly related to ethical problems between the doctor researcher and the patient or research subject; even more in countries like Peru, where the low educational level of the population increases a certain position of power of the doctor. Likewise, the appearance of new areas in Medicine such as palliative care, assisted human reproduction, gene therapy and tissue engineering make this pairing even more complex.
\end{abstract}

Key words: Medical research; Bioethics; Physician patient relationship. (source: MeSH NLM)

\section{INTRODUCCIÓN}

Aunque muchas personas piensen que cualquier tiempo pasado fue mejor, en general, la vida se ha tornado más sencilla en el tiempo. Actualmente, ya no se espera días para recibir una carta pues se cuenta con la mensajería instantánea, tampoco se requiere acudir a una biblioteca para extraer información porque casi todo está en la Internet. Esta conectividad en el mundo se debe indudablemente al desarrollo de la ciencia y la tecnología. Sin embargo, todo ese desarrollo científico tecnológico se consiguió con muchos años de investigación.
Según la Organización Mundial de la Salud, la investigación permite mejorar la salud y la calidad de vida de los individuos y las poblaciones'. Realmente la investigación en las últimas décadas ha sido de gran avance en el campo de la salud, tal es así, que la esperanza de vida de los peruanos en 1998 estaba alrededor de los 68 años y veinte años después se incrementó a 75 años $^{2}$. Este incremento se debe al desarrollo de nuevas pruebas diagnósticas y tratamientos, así como, la identificación de múltiples 
factores de riesgo de enfermedad necesarios para formular enfoques de prevención y mejorar la calidad de vida de la población. De modo tal que la investigación en salud engloba la justificación teórica pero también la importancia práctica.

Al iniciar una investigación en salud existen dos enfoques válidos: el cuantitativo y el cualitativo. El primero, permite medir y controlar ciertas variables para finalmente generalizar los resultados obtenidos, con la posibilidad de repetición y comparación entre estudios similares. El segundo, proporciona detalles sobre los datos, ilustra el entorno, y ofrece una visión más flexible. Si bien en la Medicina prevalece el enfoque cuantitativo, lo cualitativo no deja de formar parte de nuestro día a día. Y es que la relación médicopaciente se basa no sólo en cuestiones objetivas como los exámenes de laboratorio y las pruebas de imágenes sino también en cuestiones muy subjetivas como la entrevista clínica. Es en ese camino donde la Medicina nos abre un sinfín de oportunidades para las investigaciones a través de ambos enfoques que terminan siendo complementarios. Por ejemplo, al estudiar los factores determinantes en la calidad de vida de los pacientes terminales se encuentran cuestiones cuantitativas, pero además cualitativas. $Y$ son estos hechos subjetivos los que generan ese dilema ético que nos lleva a la pregunta, ¿hasta dónde se puede investigar con los seres humanos?

Hoy por hoy, la era de la medicina basada en evidencias conlleva a ofrecer el mejor tratamiento posible para los pacientes. No obstante, cuando uno se enfrenta a patologías de difícil manejo como las neoplasias en etapas avanzadas o las enfermedades raras -huérfanas- o aquellos desórdenes refractarios al tratamiento médico; en ese afán de conseguir una terapia adecuada se pueden cometer graves errores éticos, muchos de los cuales podrían quedar encubiertos bajo el nombre de alguna investigación.

\section{RELACIÓN MÉDICO PACIENTE}

La relación de un médico investigador con un sujeto de investigación sigue siendo en esencia una relación médico paciente. Algunos autores han descrito el poder en favor del médico en la relación médico paciente ${ }^{3,4}$, panorama común en países subdesarrollados o en vías de desarrollo como el Perú, donde el bajo nivel educativo de la población acrecienta cierta postura de poder del médico y relega aún más las decisiones propias del paciente. A esto se suma, el surgimiento de nuevas áreas en la Medicina como los cuidados paliativos, la reproducción humana asistida, la terapia genética y la ingeniería de tejidos, que hace aún más compleja esta relación.

Con el pasar de los años la rigurosidad científica en el mundo afectó no sólo al investigador sino también a los sujetos de investigación. Ello se constata en la relación médico paciente, esa relación paternalista de antaño donde el galeno decidía sobre el futuro de la salud del individuo viró algunas décadas atrás hacia una medicina basada en la autonomía del paciente quien actualmente exige conocer todo lo relacionado con su salud 5 . Este último tipo de relación médico paciente, es sin duda el resultado de la ética en el tiempo, la cual se basa en los principios universales descritos en el Informe Belmont de 1978: la autonomía, la beneficencia, la no maleficencia y la justicia ${ }^{6}$. La autonomía se fundamenta en el derecho a la libertad de la persona; cabe resaltar como persona autónoma aquel individuo con capacidad de discernir sobre sus intereses personales y de actuar en consecuencia a ello ${ }^{7}$. La beneficencia garantiza el bienestar y enmarca todos los actos de bondad derivados potencialmente de la investigación ${ }^{8}$. La no maleficencia explica que se debe evitar cualquier tipo de daño físico o psíquico o económico o moral a los sujetos de investigación. En tal sentido, existiría maleficencia en alguna investigación cuando el experimento contempla un riesgo mayor en comparación del beneficio que pueda recibir el sujeto de investigación ${ }^{9}$. La justicia establece una distribución equitativa de las cargas y los beneficios de la investigación entre todos los individuos participantes en el problema que se investiga. Por tanto, se debe garantizar una selección equitativa de las personas que participen en la investigación ${ }^{10}$. Estos cuatro principios universales deben tenerse en cuenta para el desarrollo de todas las investigaciones en salud y se deben plasmar en un documento: el consentimiento informado. Este documento es sumamente necesario tanto para los investigadores como para los sujetos de investigación, ya que garantiza el respeto de los sujetos de investigación participantes.

\section{CONSENTIMIENTO INFORMADO}

Aunque es evidente que los ensayos clínicos constituyen un diseño esencial de investigación para mejorar los tratamientos médicos; antes de iniciar alguno, los investigadores tienen la obligación, legal y ética, de obtener el consentimiento informado de los sujetos de investigación ${ }^{11}$.

El esbozo de consentimiento informado en el campo de la salud nació hacia 1900, cuando el doctor Walter Reed confeccionó un documento que incluía los 
riesgos y los pagos relacionados por la participación en un estudio sobre la fiebre amarilla. $Y$ aunque este texto fue más bien redactado como un contrato, se considera un documento precursor al consentimiento informado moderno ${ }^{12}$. Ya con la aparición del Código de Nüremberg en 1947 se planteó de forma explícita la obligación de solicitar el consentimiento informado, así como la libertad del participante para poner fin a cualquier experimento ${ }^{13}$.

El Colegio Americano de Médicos (ACP, por sus siglas en inglés) define al consentimiento informado como "la explicación a un paciente atento y mentalmente competente, de la naturaleza de su enfermedad, así como el balance de los efectos de esta y el riesgo de los procedimientos diagnósticos y terapéuticos recomendados, para a continuación solicitarle su aprobación para ser sometido a esos procedimientos". Asimismo, se agrega: "la presentación de la información debe ser comprensible y no sesgada (...), la colaboración del paciente debe ser conseguida sin coacción y (...) el médico no debe sacar partido de su potencial dominio psicológico sobre el paciente"14. Entonces, se debe entender más bien al consentimiento informado como un proceso gradual y continuo entre el sujeto de investigación y el investigador, que inicia con el cumplimiento del derecho a la información, se continúa con la autonomía del paciente quien debe decidir si acepta o rechaza su participación en la investigación y se culmina con el llenado apropiado del documento ${ }^{15}$. Tanto los investigadores como los sujetos de investigación son los protagonistas de este proceso. Sin embargo, la aplicación del consentimiento informado en la práctica clínica se hace notablemente difícil como señala la literatura internacional ${ }^{16}$.

Al momento de realizar el consentimiento informado existen dos perspectivas útiles: la del médico y la del paciente. La primera, permite incluir toda la información que el médico considera importante y que debe presentarse a un paciente razonable para su consentimiento informado. La segunda, más usada, por cierto, utiliza toda la información que un paciente razonable querría y necesitaría saber para tomar una elección informada. A decir verdad, hay gran confusión acerca de cuánta información se debe resumir en el consentimiento informado y de qué manera hacerlo.

Por otro lado, hay tres estilos de aprendizaje los cuales se deben considerar en el proceso del consentimiento informado. Hay sujetos de investigación que entienden y aprenden mejor visualmente, ellos quisieran ver una imagen o una fotografía o algún ejemplo demostrativo de lo que se propone. Otros entienden y aprenden mejor auditivamente, ellos preferirían escuchar con mucha atención las recomendaciones o las instrucciones pre y postoperatorias o las alternativas a la atención y los riesgos y complicaciones inherentes a algún procedimiento o tratamiento. $Y$ finalmente hay otros que son aprendices cinestésicos quienes más bien buscan relacionar cómo todo este proceso les afecta personalmente. Entonces, como el objetivo del consentimiento informado es obtener un alto nivel de comprensión, este puede lograrse al combinar los tres estilos de aprendizaje a lo largo del proceso de consentimiento informado ${ }^{17}$.

\section{NUEVOS CAMPOS DE LA MEDICINA}

La investigación en salud tiene gran interés social pues genera desarrollo y calidad de vida para las personas. En los últimos años las nuevas áreas de la Medicina han captado la atención de la sociedad, ya que ofrecen una alternativa benéfica potencial respecto a los tratamientos convencionales. Sin embargo, los dilemas éticos en investigación aumentan con el desarrollo de la ciencia y polarizan a la comunidad científica y no científica en temas como: la reproducción humana asistida y el inicio de la vida, los cuidados paliativos y el final de la vida, la terapia genética y los posibles cambios de la selección natural de la especie, el manejo in vitro de los productos biológicos de origen humano y su comercialización, la ingeniería de tejidos para posibles trasplantes de órganos, entre otros problemas; plantean diversas opiniones en favor de quienes creen que aliviar el dolor o los síntomas de una enfermedad lo justifica y otras opiniones que creen conveniente respetar los límites de la naturaleza. Lo cierto es que muchos aspectos éticos están aún por dilucidar y en el Perú hasta que no se tengan las normas claras al respecto seguirá el debate.

\section{CONCLUSIÓN}

El propósito final de la investigación médica es la producción de nuevo conocimiento para la toma de decisiones. Ese nuevo conocimiento científico es el resultado de un proceso ordenado que incluye la teoría, el método y la técnica. Por tanto, requiere de mucha responsabilidad y ética por parte del investigador o el grupo de investigación, ya que sus resultados impactan de forma directa o indirecta en la vida de la población. Lo importante antes de iniciar una investigación es tener presente que todo experimento clínico debe someterse a los principios éticos y estar dentro del marco legal, a fin de prevenir los potenciales riesgos. De forma tácita y bajo ningún criterio se puede ir en 
contra de los derechos fundamentales de los sujetos de investigación como el derecho a la vida, a la integridad física y a un trato humano. Además, una investigación debe cumplir los requisitos mínimos como el hecho de que no exista algún método alternativo eficazmente comparable al experimento, que los beneficios sean superiores a los potenciales riesgos, que el sujeto de investigación esté informado de sus derechos y que el mismo sujeto haya otorgado en forma libre su consentimiento con la posibilidad abierta en todo momento de retirarse de la investigación.

En definitiva, la ética en el marco del binomio investigación médica y práctica clínica es un desafío constante debido a los múltiples factores que afectan sus propósitos, las características de la población a estudiar, las normas socioculturales y hasta las habilidades de los investigadores. No obstante, el respeto por el paciente y el compromiso con la verdad científica debería brindar un balance necesario para conseguir este objetivo.
Contribuciones de autoría: El autor participó en la génesis de la idea, diseño de proyecto, recolección, análisis de la información y preparación del manuscrito del presente trabajo de investigación.

Financiamiento: Autofinanciado.

Conflicto de interés: El autor declara no tener conflicto de interés en la publicación de este artículo.

Recibido: 02 agosto del 2019

Aprobado: 15 septiembre del 2019

Correspondencia: Alberto Córdova Aguilar

Dirección: Jr. Paseo del Prado 133 - Urb. Las Lomas, La Molina. Lima, Perú. Teléfono: (511) 999779789

Correo:acordovaa@unmsm.edu.pe

\section{REFERENCIAS BIBLIOGRÁFICAS}

1. World Health Organization. Forty-third World Health Assembly. 1990. Disponible en: https://apps.who.int/iris/handle/10665/173422

2. Instituto Nacional de Estadística e Informática. Perú. Estimaciones y proyecciones de población por departamento, sexo y grupo quinquenales de edad 1995 - 2025. 2009. Disponible en: http://proyectos. inei.gob.pe/web/biblioineipub/bancopub/Est/Lib0846/libro.pdf

3. Borovecki A, Mlinaric A, Horvat M, Supak Smolcic V. Informed consent and ethics committee approval in laboratory medicine. Biochemia Medica. 15 de octubre de 2018;28(3):030201. Disponible en: https://doi. org/10.11613/BM.2018.030201

4. Leaning J. Human rights and medical education. BMJ. 29 de noviembre de 1997:315(7120):1390-1. Disponible en: https://doi.org/10.1136/ bmj.315.7120.1390

5. McCoy M. Autonomy, Consent, and Medical Paternalism: Legal Issues in Medical Intervention. The Journal of Alternative and Complementary Medicine. julio de 2008;14(6):785-92. Disponible en: https://doi. org/10.1089/acm.2007.0803

6. Oliva Linares JE, Bosch Salado C, Carballo Martínez R, Fernández Britto Rodríguez JE. El consentimiento informado, una necesidad de la investigación clínica en seres humanos. Revista Cubana de Investigaciones Biomédicas. junio de 2001;20(2):150-8. Disponible en: http://scielo.sld.cu/ scielo.php?script=sci_arttext\&pid=S0864-03002001000200011

7. Júdeza J, Nicolás P, Delgado MT, Hernando P, Zarco J, Granollers S. La confidencialidad en la práctica clínica: historia clínica y gestión de la información. Medicina Clínica. enero de 2002;118(1):18-37. Disponible en: https://doi.org/10.1016/S0025-7753(02)72271-9

8. Rivas García F. Límites éticos y jurídicos de la investigación biomédica. Revista de Derecho de la UNED (RDUNED). 1 de enero de 2016;0(18):293. Disponible en: https://doi.org/10.5944/rduned.18.2016.16892

9. Abajo Iglesias FJ de, Feito Grande L, Júdez Gutiérrez J, Martín Arribas MC, Terracini B, Pàmpols Ros T, et al. Directrices éticas sobre la creación y uso de registros con fines de investigación biomédica. Rev Esp Salud Publica [Internet]. febrero de 2008 [citado 4 de junio de 2019];82(1). Disponible en: http://www.scielosp.org/scielo.php?script=sci_arttext\&pid=S1135$57272008000100003 \&$ lng=es\&nrm=iso\&tlng=es
10. Abajo FJ de. La Declaración de Helsinki Vl: una revisión necesaria, pero ¿suficiente? Rev Esp Salud Publica [Internet]. octubre de 2001 [citado 4 de junio de 2019];75(5). Disponible en: http://www.scielosp.org/scielo. php?script=sci_arttext\&pid=S1135-57272001000500002\&lng=es\&nrm= iso\&tlng=es

11. Hurst SA. Declaration of Helsinki and Protection for Vulnerable Research Participants. JAMA. 26 de marzo de 2014;311(12):1252. Disponible en: 10.1001/jama.2014.1272

12. Suárez-Obando F, Ordoñez A. Ética de la Investigación científica: la fiebre amarilla, la Comisión Reed y el origen del consentimiento informado. Infectio. septiembre de 2010;14(3):206-16. Disponible en: https://doi.org/10.1016/S0123-9392(10)70112-0

13. David Pedrazas López. Aspectos éticos de la investigación biomédica: percepción de sujetos de investigación, médicos de atención primaria e investigadores de ensayos clínicos. [Barcelona]: Universitat Autónoma de Barcelona; 2016. Disponible en: https://dialnet.unirioja.es/servlet/ tesis?codigo $=117030$

14. Snyder L, American College of Physicians Ethics, Professionalism, and Human Rights Committee. American College of Physicians Ethics Manual: sixth edition. Ann Intern Med. 3 de enero de 2012;156(1 Pt 2):73-104. Disponible en: 10.7326/0003-4819-156-1-201201031-00001

15. Comisión Nacional de Bioética. Guía Nacional para la Integración y Funcionamiento de los Comités de Ética en Investigación. [Internet] 2016, 5ta ed: 16-17. [citado 4 de junio de 2019]. Disponible en: http:// www.conbioeticamexico.salud.gob.mx/descargas/pdf/registrocomites/ Guia_CEl_paginada_con_forros.pdf.

16. Chan HM. Informed Consent Hong Kong Style: An Instance of Moderate Familism. The Journal of Medicine and Philosophy. 1 de abril de 2004;29(2):195-206. Disponible en: 10.1076/jmep.29.2.195.31508

17. Haeck PC. The role of ethics in plastic surgery. En: Plastic surgery Fourth edition. London: Elsevier; 2018. p. 35-41. Disponible en: https:// www.elsevier.com/books/plastic-surgery/rubin/978-0-323-35697-8 